\section{PSICOLOGIA IBEROAMERICANA}

\section{Psicología lberoamericana}

ISSN: 1405-0943

psicología.iberoamericana@uia.mx

Universidad Iberoamericana, Ciudad de

México

México

Gaeta, Martha Leticia; Galvanovskis, Agris

Propensión a Conductas Antisociales y Delictivas en Adolescentes Mexicanos

Psicología Iberoamericana, vol. 19, núm. 2, julio-diciembre, 2011, pp. 47-54

Universidad Iberoamericana, Ciudad de México

Distrito Federal, México

Disponible en: http://www.redalyc.org/articulo.oa?id=133921440006

- Cómo citar el artículo

- Número completo

- Más información del artículo

- Página de la revista en redalyc.org

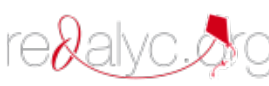

Sistema de Información Científica

Red de Revistas Científicas de América Latina, el Caribe, España y Portugal

Proyecto académico sin fines de lucro, desarrollado bajo la iniciativa de acceso abierto 


\title{
Propensión a Conductas Antisociales y Delictivas en Adolescentes Mexicanos
}

\author{
Proneness to Antisocial and Delinquent Behavior in Mexican Adolescents
}

\author{
Martha Leticia Gaeta* \\ Universidad Popular Autónoma del Estado de Puebla, México \\ Agris Galvanovskis** \\ Universidad de las Américas, Puebla, México
}

\section{RESUMEN}

Las conductas antisociales y delictivas entre los jóvenes han aumentado considerablemente en México, constituyendo una amenaza al desarrollo personal, social y económico del país. En esta investigación se buscó estudiar la propensión a estas conductas en los adolescentes, con relación al sexo, la edad y la estructura familiar. Participaron 150 estudiantes de secundaria y preparatoria, de entre 12 y 20 años de edad. Se utilizó el Cuestionario A-D, conductas antisocialesdelictivas (Seisdedos, 1995). Los resultados muestran que los hombres son más propensos que las mujeres a realizar conductas antisociales y delictivas, así como un comportamiento antisocial más agresivo. Más adolescentes que viven con un solo padre mostraron propensión a estas conductas, comparados con los que viven con ambos padres; entre 18 y 20 años mostraron más propensión a este comportamiento, que los de 12 a 14 años.

Descriptores: Comportamiento antisocial, comportamiento delictivo, adolescencia, sexo, estructura familiar.

\section{ABSTRACT}

Antisocial and delinquent behavior among young people has increased considerably in Mexico, being a threat to personal, social and economic development of the country. This investigation sought to study the proneness to this behavior in adolescents, according to gender, age and family structure. Participants were 150 students from middle and high school, between 12 and 20 years of age. The A-D, Antisocial-Delinquent Behavior Inventory (Seisdedos, 1995) was utilized. Results show that male adolescents are more prone than females to antisocial and delinquent behavior, as well as to a more aggressive antisocial behavior. More adolescents from a single parent home showed proneness to this behavior, compared to adolescents living with both parents. More adolescents 18 to 20 years old showed proneness to this behavior, than 12 to 14 years old.

Keywords: Antisocial behavior, delinquent behavior, adolescence, sex, family structure

\footnotetext{
* Para correspondencia: Doctorado en Pedagogía. Centro Interdisciplinario de Posgrados. Universidad Popular Autónoma del Estado de Puebla. 21 Sur 1103, Barrio de Santiago 72410, Puebla. Correo electrónico: marthaleticia.gaeta@upaep.mx Tel. (222) 22994 00, ext. 7781.

** Para correspondencia: Departamento de Psicología. Universidad de las Américas, Puebla. Ex hacienda Santa Catarina Mártir, 72820, San Andrés Cholula, Puebla. Correo electrónico: agris.galvanovskis@udlap.mx Tel. (222) 2292055.
} 


\section{INTRODUCCIÓN}

La adolescencia es un periodo de transformación continua que requiere ajustes a cambios biológicos, emocionales y sociales del propio desarrollo. Cuando los factores anteriores se combinan, pueden influir a que los jóvenes presenten problemas de comportamiento, particularmente conductas antisociales, y que lleguen a involucrarse en actos delictivos (Erickson, 1992).

Actualmente existe una multiplicidad de términos para referir una conducta antisocial, tales como los comportamientos agresivos e impulsivos, así como los trastornos o problemas de la conducta, entre otros (Sanabria \& Uribe, 2009). De acuerdo con Hibbs y Jensen (1996), una conducta antisocial es considerada como la oposición a las reglas de la sociedad. Esto es, una diversidad de actos que violan las normas sociales y los derechos de los demás; mientras que la delincuencia se refiere a actos cometidos por personas más jóvenes que su edad legal (Erickson, 1992). Estas desviaciones de la conducta comprenden actos legalmente definidos como delictivos, tales como violencia, robo o vandalismo, hasta una variedad de conductas no delictuosas, como agresión, peleas, prepotencia y, en general, un quebrantamiento serio de las normas en el hogar y en la escuela (Kazdin, 1988; Erickson, 1992).

Las conductas antisociales a menudo tienen serias consecuencias inmediatas, tanto para el joven que las tiene (por ejemplo, expulsión de la escuela u hospitalización) como para aquellos con quienes interactúa (por ejemplo, las víctimas). Además, al llegar a adultos, estos jóvenes están en riesgo de diversos problemas personales y sociales, tanto en el trabajo como en el hogar $y$, en ocasiones, de conducta delictiva y alcoholismo (Kazdin, 1988). La delincuencia implica altos costos económicos y sociales por la desintegración de las familias, la pérdida de vidas, la atención de la salud, así como programas educativos y de rehabilitación (Sanabria \& Uribe, 2009). Por lo que la participación de los jóvenes en actos antisociales y delictivos es considerada como una amenaza potencial para el desarrollo personal, social y económico de un país (Organización Mundial de la Salud, OMS, 2003).

En México, las conductas antisociales, y en especial la delincuencia, han aumentado en los últimos años. Un estudio llevado a cabo en la Ciudad de México con más de 10000 estudiantes de nivel medio y medio superior reveló un incremento en este tipo de comportamiento en los jóvenes, y la tasa fue mayor en las mediciones hechas entre 2000 y 2003. En general, se incrementaron en mayor medida las conductas relacionadas con el factor de violencia y robos -que incluía actos como tomar dinero con valor menor a 50 pesos, tomar mercancía sin pagar, tomar parte en riñas, golpear o dañar objetos, golpear o herir a personas y prender fuego a objetos-, y en menor grado los actos antisociales graves -tomar valores por 500 pesos o más, forzar cerraduras, atacar a alguien usando algún objeto o arma, vender drogas y usar un cuchillo o pistola para robar (Medina-Mora, Villatoro, Gutiérrez, Juárez \& Fleiz, 2005)-. Así también, estadísticas del Consejo de Menores indican que en el Distrito Federal el número de jóvenes puestos a disposición aumentó de 2556 en 1998 a 4166 en 2005, y en el ámbito nacional, tan sólo durante 2002, ingresaron más de $31 \mathrm{mil}$ adolescentes de entre 11 y 17 años de edad a centros de tratamiento juveniles. Cifras que ponen de manifiesto la necesidad de analizar la evolución de dichos comportamientos, así como los factores que propician este tipo de conductas entre los jóvenes (Secretaría de Seguridad Pública, 2005).

Desde este enfoque, investigadores en diferentes países han encontrado que el comportamiento antisocial y delictivo tiene un inicio temprano en los jóvenes, y la violencia más seria tiende a aumentar con la edad, acentuándose especialmente durante la adolescencia (Loeber \& Hay, 1997; Storvoll \& Luichstrom, 2003). Así, en un estudio con 4152 adolescentes españoles entre 12 y 17 años de edad, se encontró que éstos comenzaban a presentar estos comportamientos a partir de los 13 años de edad. Además, 9.2\% de ellos se consideran "delincuentes peligrosos" (Rechea, 2008). Otra investigación con adolescentes colombianos (Sanabria \& Uribe, 2009) reveló que los jóvenes entre 16 y 18 años de edad eran los que más mostraban conductas antisociales y delictivas, en comparación con los de 12 a 13 años de edad. Un estudio similar en Estados Unidos mostró que los chicos entre 8 y 14 años de edad tenían relativamente leves problemas, y los de 15 a 20 años más severos en cuanto al uso de alcohol y marihuana, historia delictiva por violencia y delitos por daños a propiedad y desorden público (Dembo, Schmeidler, Nini-Gough \& Manning, 1998). En un 
estudio en México, se observó que los jóvenes de 18 años de edad cometieron un mayor número de delitos y actos antisociales en general, que los de 15 años de edad o menos (Juárez et al., 1998). Por su parte, una investigación con adolescentes mujeres norteamericanas (Talbott \& Thiede, 1999) demostró cierta continuidad de actos antisociales en el tiempo, relacionados con actos destructivos, vandalismo y peleas, no así con el consumo de alcohol, robos y problemas escolares.

Por otro lado, investigaciones recientes han indicado que el comportamiento antisocial difiere en hombres y mujeres en forma, frecuencia y variabilidad (Storvoll \& Luichstrom, 2003). Así, las manifestaciones de agresión a través de la niñez, adolescencia y edad adulta temprana no son las mismas para cada género (Loeber \& Hay, 1997). Si bien es cierto que estos problemas de conducta se incrementan en la adolescencia, tanto para los hombres como para las mujeres, la investigación ha demostrado que el aumento es más marcado en las mujeres. En particular, los problemas de conducta no agresivos físicamente, asociados a las mujeres son: expandir rumores o fomentar el rechazo de otros hacia la víctima (Conway, 2005).

Aunado a lo anterior, independientemente de la cultura o época, los hombres son más agresivos que las mujeres, y en la adolescencia son responsables de más actos delictivos (Rechea, 2008; Sanabria \& Uribe, 2009). Un estudio en Estados Unidos reveló que los adolescentes hombres en edad escolar secundaria mostraron más conductas antisociales y delictivas que las adolescentes (Flannery, Williams \& Vazsonyl, 1999). Asimismo, otros estudios con adolescentes norteamericanos (Liu \& Kaplan, 1999) y adolescentes chinos (Ma, 2003) corroboraron que los hombres, comparados con las mujeres, eran más antisociales en sus conductas y presentaban niveles más altos de comportamientos delictivos. Una investigación con adolescentes mexicanos reveló que, en promedio, los estudiantes hombres, en comparación con las mujeres, estaban involucrados en un mayor número de actos antisociales y estas conductas eran más severas bajo el consumo de alcohol o drogas (Juárez et al., 1998).

Como se observa, existe un número considerable de investigaciones sobre conductas antisociales y delictivas en función de la edad y el sexo. Sin embargo, la mayoría de estos estudios se ha realizado en otros países. En este sentido, Kazdin (1988) afirma que el punto de referencia para una conducta antisocial siempre es el contexto sociocultural en que surge. Específicamente, un factor asociado significativamente con las conductas antisociales es la estructura familiar (Simon, Wei, Conger \& Elder, 2001). De ahí que en la presente investigación se pretenda evaluar la propensión de adolescentes mexicanos, hombres y mujeres de diferentes edades, a realizar conductas antisociales y delictivas. Además de establecer una relación de estos comportamientos, considerando la estructura familiar de los jóvenes.

\section{MÉTODO}

\section{Participantes}

Participaron 150 estudiantes de secundaria y preparatoria de un colegio privado en la ciudad de Puebla, México. Del total de adolescentes, 77 eran hombres y 73 mujeres. La edad promedio de la muestra era de 15.85 años (D.E. $=2.37$ años). $36 \%$ de los adolescentes se encontraba entre los 12 y 14 años; $30 \%$ entre los 15 y 17 años; y $34 \%$ entre 18 y 20 años. El método de selección de la muestra fue por disponibilidad.

\section{Instrumento}

La propensión a realizar conductas antisociales y delictivas se midió con el Cuestionario $A-D$, conductas antisociales-delictivas, desarrollado por Seisdedos (1995), con la adaptación a la población mexicana de Seisdedos y Sánchez (2001). La escala evalúa los aspectos antisocial y delictivo de la conducta desviada en niños y adolescentes. Los jóvenes contestaron si habían realizado alguna vez el comportamiento particular ( $\mathrm{si}=1 \mathrm{o}$ no $=0$ puntos), de un total de 40 reactivos ubicados en dos factores (20 elementos de la escala A - conductas antisociales, y 20 de la escala $\mathrm{D}$ - conductas delictivas).

La escala $A$ - conductas antisociales se refiere a comportamientos no expresamente delictivos, aunque sí desviados de las normas y usos sociales considerados deseables. Incluye conductas como "ensuciar las calles y aceras rompiendo botellas o volcando cubos de basura", "hacer desórdenes en lugares públicos", "gastar bromas pesadas a otra persona".

La escala $D$ - conductas delictivas incluye comportamientos que suelen estar fuera de la ley como 
"robar cosas de los coches", "llevar algún arma como cuchillo o navaja por si es necesario en una pelea", "conseguir dinero amenazando a personas más débiles".

Cada uno de 40 reactivos obtenidos en la escala final es el resultado del análisis factorial de 137 reactivos iniciales, con una muestra de 405 hombres y 604 mujeres, con una confiabilidad ( $\alpha$ de Cronbach) en las conductas antisociales de .866 para los hombres y .860 para las mujeres, así como de .862 para hombres y .860 para mujeres en las conductas delictivas. El cuestionario cuenta con un índice de confiabilidad ( $\alpha$ de Cronbach) de .88 para cada una de las escalas.

Aunado a lo anterior, se preguntó a los estudiantes, al inicio del cuestionario, su edad, sexo y si vivían con ambos padres o con uno.

\section{PROCEDIMIENTO}

Los cuestionarios fueron aplicados grupalmente en el salón de clases durante el horario escolar. Se enfatizó la importancia de contestar con sinceridad a la totalidad de las preguntas. Asimismo, se aseguró la confidencialidad de la información a los participantes (los alumnos no tuvieron que indicar su nombre en los cuestionarios).
La estadística inferencial, la prueba $t$ de Student y el análisis de varianza fueron utilizados para el tratamiento de datos.

\section{RESULTADOS}

En las tablas que siguen ( 1 y 2 2) se presentan las características generales de la muestra. Los porcentajes presentados se refieren a la proporción de cada categoría de adolescentes con respecto al número total de participantes.

Las variaciones entre los adolescentes respecto a la edad y al sexo se presentan en la Tabla 1. Como se observa, participó casi igual número de hombres y mujeres en los grupos de diferentes edades. También estuvieron representados en forma equitativa en todos los grupos por edad.

En la Tabla 2 se presentan los datos referentes a la estructura familiar de los adolescentes. Se observa que un mayor número de jóvenes vive en familias con ambos padres $(n=133)$, mientras que un menor número $(n=17)$ provienen de familias con un solo padre. Esto es, la mayoría de los adolescentes vive con ambos padres (88.7\%). Además, el grupo de 12 a 14años de edad presenta un mayor número de familias con ambos padres.

Tabla 1. Estructura de la muestra por edad y sexo

\begin{tabular}{|c|c|c|c|c|c|c|}
\hline Variable & \multicolumn{6}{c|}{ Edad } \\
\hline \multicolumn{2}{|c|}{12 a 14 años } & \multicolumn{2}{c|}{15 a 17 años } & 18 a 20 años \\
\hline Sexo & $\mathrm{N}$ & $\%$ & $\mathrm{~N}$ & $\%$ & $\mathrm{~N}$ & $\%$ \\
\hline Hombres & 28 & 18.7 & 26 & 17.3 & 23 & 15.3 \\
\hline Mujeres & 26 & 17.3 & 19 & 12.7 & 28 & 18.7 \\
\hline
\end{tabular}

Tabla 2. Composición de la muestra por edad y estructura familiar

\begin{tabular}{|c|c|c|c|c|c|c|}
\hline Variable & \multicolumn{2}{c|}{ Edad } \\
\hline \multicolumn{2}{|c|}{12 a 14 años } & \multicolumn{2}{c|}{15 a 17 años } & 18 a 20 años \\
\hline Padres & $N$ & $\%$ & $N$ & $\%$ & $N$ & $\%$ \\
\hline Ambos & 52 & 34.7 & 37 & 24.7 & 44 & 29.3 \\
\hline Un padre & 2 & 1.3 & 8 & 5.3 & 7 & 4.7 \\
\hline
\end{tabular}




\section{Diferencias por sexo}

A fin de buscar las diferencias en conductas antisociales y delictivas por sexo se utilizó la prueba $t$ de Student para muestras independientes. En la Tabla 3 se observa que existen diferencias estadísticamente significativas entre los hombres y las mujeres, tanto en conductas antisociales $(t=2.83 ; p<.01)$ como en conductas delictivas $(t=5.48 ; p<.01)$. lictivas entre diferentes grupos de edad: a) $12-14, b$ ) $15-17$ y c) $18-20$ años.

Se observaron diferencias estadísticamente significativas entre los grupos en las conductas antisociales $(F=11.81 ; p<0.001)$ y las conductas delictivas $(F=5.42$; $p<0.01)$.

Se encontraron diferencias significativas entre los grupos a) $12-14$ y c) $18-20$, tanto en conductas an-

Tabla 3. Diferencias en conductas antisociales y delictivas por sexo

\begin{tabular}{|c|c|c|c|c|c|}
\hline \multirow[t]{3}{*}{ Variable } & \multicolumn{4}{|c|}{ Sexo } & \\
\hline & \multicolumn{2}{|c|}{$\begin{array}{c}\text { Hombres } \\
(n=77)\end{array}$} & \multicolumn{2}{|c|}{$\frac{\text { Mujeres }}{(n=73)}$} & \\
\hline & Media & $\mathrm{DE}$ & Media & DE & $t$ \\
\hline Conductas antisociales & 12.16 & 4.82 & 10.08 & 4.17 & $2.83^{*}$ \\
\hline Conductas delictivas & 3.29 & 3.84 & 0.68 & 1.32 & $5.48^{*}$ \\
\hline
\end{tabular}

${ }^{*} p<0.01$

En cuanto a las conductas antisociales, más hombres que mujeres admitió haber roto botellas o volcado cubos de basura en la calle $(t=4.15 ; p<.0 \mathrm{o} 1)$, molestado a desconocidos, hecho desórdenes en lugares públicos $(t=3.21 ; p<.01)$, roto cosas de terceros $(t=2.79 ; p<.01)$ o gastado bromas pesadas a otra persona $(t=5.09$; $p<.001)$, contestado mal a un superior $(t=1.99 ; p<.05)$ o haberse peleado con otros $(t=6.51 ; p<.001)$. Por su parte, un mayor número de mujeres, en comparación con los hombres, reportó haber hecho trampas -en examen, competición importante o información de resultados- $(t=2.66 ; p<.01)$ y llegado tarde al colegio o reunión $(t=2.02 ; p<.05)$.

Respecto a las conductas delictivas, más hombres que mujeres reportaron haber pertenecido a una pandilla $(t=2.12 ; p<.05)$, portado cuchillo o navaja $(t=3.65$; $p<.001)$, forcejeado con un policía $(t=2.24 ; p<.01)$, destrozado cosas en lugares públicos $(t=4.66 ; p<.001)$, realizado robos $(t=4.00 ; p<.001)$ y comprado bebidas prohibidas $(t=4.89 ; p<.001)$.

\section{Diferencias por edad}

El análisis de varianza unidireccional fue utilizado para ver las diferencias en conductas antisociales y de- tisociales $(p<0.001)$ como delictivas $(p<0.01)$. Esto es, un mayor número de adolescentes en edad entre $18 \mathrm{y}$ 20 años reportó haberse involucrado en más actos antisociales y delictivos, en comparación con los de 14 años y menos. No existen diferencias significativas entre el número y la propensión a conductas antisociales y delictivas de los adolescentes de 15 a 17 y de 18 a 20 años de edad. Entre los grupos a) 12 - 14 y $b) 15-17$ se encontraron diferencias significativas en las conductas antisociales $(p<0.001)$, pero no así en las delictivas.

\section{DISCUSIÓN}

Los resultados ponen de manifiesto la existencia de diferencias significativas entre los diferentes grupos estudiados (en función del sexo y la edad). De manera específica, los hallazgos del presente estudio indican que los adolescentes hombres son más propensos que las mujeres a realizar conductas antisociales y delictivas, cuando el ambiente y las circunstancias lo favorecen. Además, ellos presentan más conductas antisociales agresivas que ellas.

Los resultados anteriores son consistentes con los de estudios similares, tanto en otros países como en el nuestro (Flannery et al., 1999; Juárez et al., 1998; Liu \& 
Kaplan, 1999; Ma, 2003). Esto, en general, induce a pensar que los hombres, comparados con las mujeres, están en mayor riesgo de realizar actos antisociales y tienen mayor tendencia a involucrarse en actos delictivos.

En el presente estudio, un mayor número de hombres, en comparación con las mujeres, admitió haber realizado conductas agresivas, como pelearse con otros (con golpes, insultos o palabras ofensivas). Más mujeres, por su parte, indicaron hacer trampas (en examen o información de resultados) y llegar tarde al colegio o reunión. Estos resultados concuerdan con la opinión de otros investigadores (Sanabria \& Uribe, 2009), en el sentido de que las mujeres tienden a involucrarse en más comportamientos antisociales no agresivos y los hombres tienden a ser más agresivos en sus conductas. También es posible que los patrones educacionales de nuestro país (los hombres deben ser valientes, fuertes y agresivos) determinan que ellos manifiesten un comportamiento antisocial más agresivo que las mujeres (Juárez et al., 1998).

Es importante señalar que, aunque en menor grado, las mujeres admitieron algunas conductas antisociales efectuadas por los hombres, lo cual coincide con la investigación de Conway (2005) en lo referente a que en los hombres, más que en las mujeres, los problemas de conducta se incrementan en el periodo de la adolescencia. No obstante, la investigación y los datos de este estudio apuntan hacia que, independientemente del sexo, una buena parte de los adolescentes se involucra en ciertos problemas de comportamiento antisocial (Medina-Mora et al., 2005).

Otro factor asociado de manera importante con las conductas antisociales es la estructura familiar (Simon et al., 2001). Cabe mencionar que los participantes de esta investigación son, en su mayoría, de familias con ambos padres (alrededor de 89\%). En este sentido, existe evidencia que, más que la estructura, el medio ambiente familiar (las relaciones interpersonales) y la supervisión de los padres pueden alentar o ayudar a disminuir las conductas antisociales en los jóvenes (Carson, Chowdhury, Perry \& Pati, 1999). Por lo que resulta necesario analizar más a fondo las relaciones en las diferentes estructuras familiares para con estas conductas en futuras investigaciones.

Respecto a la edad, un mayor número de adolescentes de entre 18 y 20 años de edad muestra pro- pensión a realizar conductas antisociales y delictivas, comparados con adolescentes de 14 años y menos. Sin embargo, no existen diferencias significativas entre el número y la propensión de los adolescentes de 15 a 17 y de 18 a 20 años. Entre los adolescentes de 12 a 14 y 15 a 17 años hay diferencias significativas en la propensión a conductas antisociales, pero no así a conductas delictivas.

Las diferencias significativas en la propensión a conductas antisociales y delictivas encontradas entre los grupos de jóvenes en los inicios y finales de la adolescencia son similares a los resultados de otros estudios (Juárez et al., 1998; Sanabria \& Uribe, 2009). Estos datos inducen a pensar que, si un adolescente muestra propensión hacia estas conductas al inicio de la adolescencia, existe el riesgo de escalar hacia un comportamiento más problemático y delictivo hacia el final de esta etapa y edad adulta temprana; además de presentar un alto riesgo de involucrarse en otros problemas como dificultades académicas, consumo de sustancias psicoactivas y comportamientos sexuales de riesgo (Sanabria \& Uribe, 2009).

Los resultados de este estudio indican, asimismo, diferencias en la propensión a conductas antisociales y delictivas entre los y las adolescentes de diferentes edades. Esto implica que es necesario considerar a cada adolescente de acuerdo a su sexo y etapa de desarrollo al analizar estas conductas.

Finalmente, es importante señalar que, teniendo en cuenta el tamaño de la muestra, el presente trabajo de investigación es exploratorio $y$, por lo tanto, no podemos generalizar los resultados a la población de adolescentes mexicanos. Todo ello da lugar a futuras investigaciones con una mayor muestra que confirmen los resultados obtenidos.

En general, los resultados sugieren la necesidad de desarrollar programas preventivos (Garaigordobil, 2004), de forma que, una vez detectada la propensión hacia conductas antisociales en los jóvenes, ésta no desemboque en problemas de comportamiento mayores. Tanto padres como maestros, quienes son los principales responsables de la prevención de estos comportamientos, deberían trabajar juntos a fin de preparar a los adolescentes para adquirir las habilidades sociales que los hagan superar esta etapa del desarrollo con éxito. Esto va asociado a tener un mayor acercamiento 
con ellos y darles apoyo individualizado, ya que cada persona experimenta un grado diferente de vulnerabilidad hacia las conductas antisociales y delictivas.

\section{REFERENCIAS}

Carson, D. K., Chowdhury, A., Perry, C. K. y Pati, Ch. (1999). Family characteristics and adolescent competence in India: Investigation of youth in Southern Orissa. Journal of Youth and Adolescence, 28(2), 211233.

Conway, A. M. (2005). Girls, Aggression and Emotion Regulation. American Journal of Orthopsychiatry, 75(2), 334-339.

Dembo, R., Schmeidler, J., Nini-Gough, B. y Manning, D. (1998). Sociodemographic, delinquency-abuse history, and psychosocial functioning differences among juvenile offenders of various ages. Journal of Child and Adolescent Substance Abuse, 8(2), 63-78.

Erickson, M. T. (1992). Behavior disorders of children and adolescents. New Jersey: Prentice Hall, Inc.

Secretaría de Seguridad Pública. Estadísticas del Consejo de Menores del Distrito Federal. (2005). México, D.F. Recuperado de: http://www.ssp.gob. $\mathrm{mx} /$ portalWebApp/ShowBinary?nodeId=/BEA $\% 20$ Repository/99095//archivo

Flannery, D. J., Williams, L. L. y Vazsonyl, A. T. (1999). Who are they with and what are they doing? Delinquent behavior, substance use, and early adolescents' after school time. American Journal of Orthopsychiatry, 69(2), 247-253.

Garaigordobil, M. (2004). Intervención psicológica en la conducta agresiva y antisocial con niños. Psicothema, 16(3), 429-435.

Hibbs, E. D. y Jensen, P. S. (1996). Psychosocial treatments for child and adolescent disorders. Washington, D. C.: American Psychological Association.

Juárez, F., Medina-Mora, E., Berenzon, S., Villatoro, J. A., Carreño, S., López, E. K., Galvan, J., y Rojas, E. (1998). Antisocial behavior. Its relation to selected sociodemographic variables and alcohol and drug use among Mexican students. Substance Use and Misuse, 33(7), 1437-1459.
Kazdin, A. E. (1988). Tratamiento de la conducta antisocial en la infancia y la adolescencia. Barcelona: Martínez Roca, S. A.

Liu, X. y Kaplan, H. B. (1999). Explaining the gender difference in adolescent delinquent behavior: a longitudinal test of mediating mechanisms. Criminology, 37(1), 195-215.

Loeber, R. y Hay, D. (1997). Key issues in the development of aggression and violence from childhood to early adulthood. Annual Review of Psychology, 48, 371-411.

Ma, H. K. (2003). The relation of moral orientation and moral judgment to prosocial and antisocial behavior of Chinese adolescents. International Journal of Psychology, 38(2), 101-111.

Medina-Mora, M., Villatoro, V., Gutiérrez, M., Juárez, F. y Fleiz, C. (2005). Tendencias de la conducta antisocial en estudiantes del Distrito Federal: Mediciones 1997-2003. Salud Mental, 28(3), 60-68.

Organización Mundial de la Salud (2003). Informe mundial sobre la violencia y la salud. Washington: OMS.

Rechea, C. (2008). Conductas antisociales y delictivas de los jóvenes en España. Castilla-La Mancha: Centro de Investigación en Criminología de la Universidad de Castilla-La Mancha. Difundido por el Consejo General del Poder Judicial (CGPJ).

Sanabria, A. M. y Uribe, A. F. (2009). Conductas antisociales y delictivas en adolescentes infractores y no infractores. Pensamiento Psicológico, 6(13), 203-218.

Seisdedos, C. N. (1995). Cuestionario A-D, conductas antisociales-delictivas. (2 $2^{\text {a }}$ ed.). Madrid: TEA Ediciones, S. A.

Seisdedos, C. N., y Sánchez, P. (2001). Cuestionario de conductas antisociales-delictivas A-D. México: El Manual Moderno.

Simon, R., Wei, Ch., Conger, R. y Elder, G. (2001). Quality of parenting as mediator of the effect of childhood 
defiance on adolescent friendship choices and delinquency: a growth curve analysis. Journal of Marriage and Family, 63(1), 63-80.

Storvoll, E. y Luichstrom, L. (2003). Gender differences in changes and stability of conduct problems from early adolescence to early adulthood. Journal of Adolescence, 26(4), 413-430.

Talbott, E. y Thiede, K. (1999). Pathways to antisocial behavior among adolescent girls. Journal of Emotional and Behavioral Disorders, 7(1), 31-43. 